\title{
Bronchoscopic Coil Treatment for Patients with Severe Emphysema: A Meta-Analysis
}

\author{
Dirk-Jan Slebos ${ }^{a}$ Jorine E. Hartman ${ }^{a}$ Karin Klooster ${ }^{a}$ Stefan Blaas ${ }^{b}$ Gaetan Deslee $^{\mathrm{i}}$ \\ Wolfgang Gesierich $^{c}$ Juergen Hetzel ${ }^{d} \quad$ Martin Hetzel $^{\mathrm{e}}$ William McNulty ${ }^{\mathrm{l}, \mathrm{m}}$ \\ Samuel V. Kemp ${ }^{1, m}$ Romain Kessler ${ }^{j}$ Sylvie Leroy ${ }^{k}$ Franz Stanzel ${ }^{f}$ Christian Witt $^{g}$ \\ Zaid Zoumot, ${ }^{1, n}$ Felix J.F. Herth ${ }^{h}$ Pallav L. Shah',m \\ ${ }^{a}$ Department of Pulmonary Diseases, University Medical Center Groningen, University of Groningen, Groningen, \\ The Netherlands; ${ }^{b}$ Center for Pneumology, Donaustauf Hospital, Donaustauf, ' Comprehensive Pneumology \\ Center Munich, Asklepios Fachkliniken München-Gauting, Gauting, dUniversity Hospital Tübingen, Tübingen, \\ e Krankenhaus vom Roten Kreuz Bad Cannstatt, Stuttgart, ${ }^{\mathrm{f}}$ Lungenklinik Hemer, Hemer, ${ }^{\mathrm{g}}$ Campus Charité Mitte, \\ Berlin, and hepartment of Pneumology and Critical Care Medicine, Thoraxklinik, Translational Lung Research \\ Center Heidelberg, University of Heidelberg, Heidelberg, Germany; ' Department of Pulmonary Medicine, University \\ Hospital of Reims, Reims, 'ंDepartment of Pulmonary Medicine, University Hospital of Strasbourg, Strasbourg, and \\ kDepartment of Pulmonary Medicine, University Hospital of Nice, Nice, France; 'The National Institute for Health \\ Research Respiratory Biomedical Research Unit, Royal Brompton and Harefield NHS Foundation Trust and \\ Imperial College, and ${ }^{\mathrm{m}}$ Chelsea and Westminster Hospital, London, UK; ${ }^{\mathrm{n}}$ Cleveland Clinic Abu Dhabi, Abu Dhabi, \\ United Arab Emirates
}

\section{Key Words}

Chronic obstructive pulmonary disease - Emphysema .

Bronchoscopic lung volume reduction

\begin{abstract}
Background: Bronchoscopic coil treatment has been shown to improve pulmonary function, exercise capacity, and quality of life in patients with severe emphysema. Objectives: To perform a meta-analysis of the results of four independent European clinical trials investigating this coil therapy for emphysema. Methods: Data on all patients included in the four European clinical trials were analyzed for efficacy and safety outcomes. Results: A total of 2,536 coils were placed during 259 procedures in 140 patients. A total of 37 chronic obstructive pulmonary disease exacerbations and 27 pneumonias were recorded as serious adverse events up to 1 year after
\end{abstract}

treatment. The pneumothorax rate was $6.4 \%$. Both 6 and 12 months after treatment, significant (all $p<0.001$ ) improvements were observed for: forced expiratory volume in $1 \mathrm{~s}$ $[+0.08$ liters $( \pm 0.19)$ and +0.08 liters $( \pm 0.21)]$, residual volume $[\mathrm{RV} ;-510 \mathrm{ml}( \pm 850)$ and $-430 \mathrm{ml}( \pm 720)], 6$-min walking distance $[6 \mathrm{MWD} ;+44.1 \mathrm{~m}( \pm 69.8)$ and $+38.1 \mathrm{~m}( \pm 71.9)]$, and St. George's Respiratory Questionnaire score [SGRQ; -9.5 points $( \pm 14.3)$ and -7.7 points $( \pm 14.2)$ ]. No differences in any outcome measures were observed between heterogeneous and homogeneous emphysema patients. Only a high baseline RV was found to be an independent predictor of successful treatment. Conclusions: Bronchoscopic coil treatment improves pulmonary function, 6MWD, and quality of life in patients with severe emphysema up to 1 year after treatment, independent of the distribution of the disease.

(c) 2015 S. Karger AG, Basel

\section{KARGER 125}

(c) 2015 S. Karger AG, Base

0025-7931/15/0902-0136\$39.50/0 


\section{Introduction}

Bronchoscopic lung volume reduction treatment with nitinol coils has been intensively investigated in recent years as a treatment option for patients with severe emphysema. The first in-man trial placing up to 5 first-generation coils showed their safety and feasibility in patients with severe homogeneous and heterogeneous emphysema [1]. This safety study met its endpoint. After this trial, the coil treatment was optimized in a pilot study by placing around 10 coils per upper lobe $(n=16)$ [2], showing significant improvements in pulmonary function testing, 6-min walking distance (6MWD), and quality of life questionnaires in patients with upper lobe disease. A 6-month responder rate between 60 and $70 \%$ of patients achieving or exceeding the minimal clinically important difference (MCID) for forced expiratory volume in $1 \mathrm{~s}\left(\mathrm{FEV}_{1}\right)$ [3], residual volume (RV) [4], 6MWD [5], and St. George's Respiratory Questionnaire (SGRQ) [6] score was documented. These findings were repeated in a randomized controlled trial $(n=46)$ [7] and in a European multicenter feasibility trial $(n=60)$ [8]. A small prospective singlecenter trial investigating only homogeneous patients showed similar improvements, with about $70 \%$ of the patients at or above the MCID for the same endpoints [9].

Despite very similar baseline characteristics and inclusion/exclusion criteria, no best responder profile emerged within each individual trial data set. The small size of each study prevented firm conclusions; however, the consistency of the protocol designs presents an opportunity to analyze composite raw data with a stronger statistical power.

In the current study, we performed a meta-analysis of the raw data from all four clinical trials that have been performed using the same treatment algorithm (bilateral treatment placing up to 12 coils per lobe) and coil design (second-generation RePneu ${ }^{\circledR}$ endobronchial coil).

\section{Subjects and Methods}

This meta-analysis was performed using the raw original individual patient data for all patients included in four lung volume reduction coil trials: ClinicalTrials.gov registry No. NCT01220908 [2], NCT01334307 [7], NCT01421082 [9], and NCT01328899 [8]. From the RESET trial (NCT01334307) [7], also the 12-month follow-up and data on patients after crossover were included [10]. From the CLN0006 study (NCT01220908), 9 extra patients were included in this meta-analysis who were treated outside the Netherlands and not included in the original Dutch study [2]. These trials have been performed in a total of 13 different hospitals throughout Europe. The first patient from these four trials was treated in April 2009, the last in September 2012. All patients participating in these trials gave written informed consent, and all trials were approved by the local ethics committees.

\section{Patients}

The inclusion and exclusion criteria were very similar across all four trials, evaluating RePneu coil treatment in severe chronic obstructive pulmonary disease (COPD) patients with emphysema as evidenced on a chest CT scan. The main inclusion and exclusion criteria for these four trials are presented in table 1.

\section{Lung Volume Reduction Coil Treatment}

The endobronchial coil treatment was performed as previously described [2]. In brief, the RePneu coil (PneumRx Inc., Mountain View, Calif., USA; fig. 1a) is an implantable, preformed, coil-shaped device composed of nitinol wire. The coil is placed in the lung tissue using a flexible therapeutic bronchoscope (working channel $\geq 2.8 \mathrm{~mm}$ ). Before placement, a dedicated guide wire is used to guide the delivery catheter to the subsegmental airway and to measure the length of the coil needed. Using a dedicated delivery system including a specialized forceps, the coil is loaded into a cartridge which straightens the coil for delivery via the catheter into the subsegmental airways. The position of the coil is confirmed under fluoroscopic guidance and released by easing the coil forward and retracting the delivery catheter. Once deployed, the coil recovers to its predetermined shape. Seven sizes of coil were available for use in the four trials $(70,85,100,125,150,175$, and $200 \mathrm{~mm})$; however, in $96 \%$ of the procedures, only the $100-, 125-$, or 150 -mm coils were used. The coil procedures were performed either using moderate sedation or general anesthesia. Per protocol the most diseased lobe in each lung was treated in two separate bronchoscopic procedures, with the second procedure at least 1 month after the first procedure.

\section{Assessments and Follow-Up}

Both baseline and follow-up assessments were similar in all trials. At baseline, documentation of medication and the medical history were examined, and a physical examination, dyspnea assessment by the modified Medical Research Council (mMRC) scale [11], chest CT scan, and echocardiogram were performed. Pulmonary function at baseline was measured by post-bronchodilator spirometry, body plethysmography, and measurement of diffusion capacity, all performed according to the current ATS/ERS guidelines $[12,13]$. Exercise capacity was assessed using the 6-min walk test according to the ATS guidelines [14], and quality of life was measured using the SGRQ [15]. At follow-up, a general health assessment, documentation of adverse events, physical examination, mMRC dyspnea score assessment, post-bronchodilator spirometry and body plethysmography, 6-min walk test, and the SGRQ were performed.

In all studies, patients were followed up until 12 months after the coil treatment, except for 23 German patients in the European multicenter trial [8], who exited the trial at 6 months per protocol. For the meta-analysis, we collected all data at baseline, 6 months, and 12 months after treatment.

The primary efficacy endpoint for three of the four studies [2, 7,8 ] was improvement in SGRQ total score from baseline, and in one study [9], the primary endpoint was improvement in 6MWD. The safety objectives in all studies were to identify the number and type of device-related and procedure-related adverse events associated with the use of endobronchial coils. 
Fig. 1. a The RePneu coil in its original shape (the actual total wire length shown is $125 \mathrm{~mm}$; used with permission of Pneum$\mathrm{Rx})$. b X-ray image after bilateral upper lobe coil treatment.
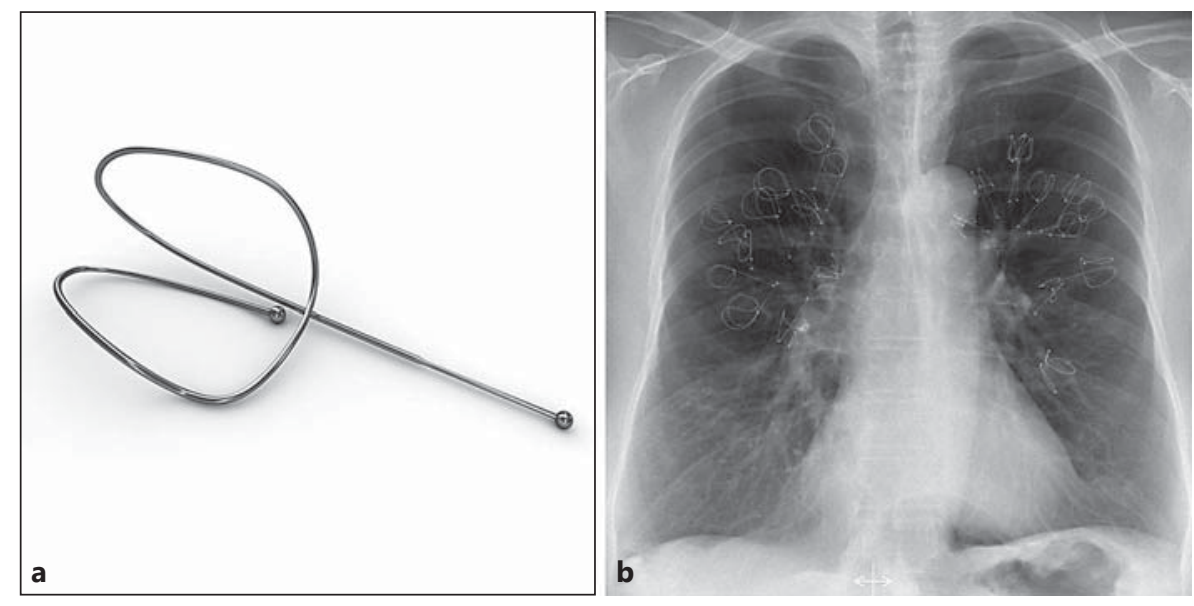

Table 1. Inclusion and exclusion criteria of the four trials included in this meta-analysis

$\begin{array}{llll}\begin{array}{l}\text { Slebos et al. [2] } \\ (\mathrm{n}=16)\end{array} & \begin{array}{l}\text { Shah and colleagues [7,10] } \\ (\mathrm{n}=45)\end{array} & \begin{array}{l}\text { Klooster et al. [9] } \\ (\mathrm{n}=10)\end{array} & \begin{array}{l}\text { Deslee et al. [8] } \\ (\mathrm{n}=60)\end{array}\end{array}$

Major inclusion criteria

Emphysema distribution on chest CT

(physician's judgment)

Age, years

$\mathrm{FEV}_{1}, \%$ predicted

TLC, $\%$ predicted

$\mathrm{RV}, \%$ predicted

mMRC score

Stopped smoking, weeks prior to study

\section{Heterogeneous upper lobe}

$\begin{array}{ll} & \text { homogeneous distribution } \\ \geq 35 & \geq 35 \\ \leq 45 & \leq 45 \\ >100 & >100 \\ - & - \\ \geq 2 & \geq 2 \\ >8 & >8\end{array}$

Homogeneous distribution

\section{$\geq 35$}

$\leq 45$

$>100$

$>225$

$\geq 2$

$>26$
Heterogeneous upper and lower lobe

$\geq 35$

$\leq 45$

$>100$

$>175$

$\geq 2$

$>8$

Major exclusion criteria (similar for all studies)

$\mathrm{PaCO}_{2}>8.0 \mathrm{kPa}$ or $\mathrm{PaO}_{2}<6.0 \mathrm{kPa}$

Post-bronchodilator $\mathrm{FEV}_{1}$ change $>20 \%$

DLCO $<20 \%$ of predicted

6 MWD $<140 \mathrm{~m}$

Recurrent respiratory infections

Pulmonary hypertension (RSVP $>50 \mathrm{~mm} \mathrm{Hg}$ on echocardiogram)

Giant bullae occupying $>1 / 3$ of the lung

Previous lung volume reduction surgery, lobectomy, or pneumonectomy

Congestive heart failure (LVEF $<45 \%$ on echocardiogram)

Warfarin, coumarin, or clopidogrel treatment

Other disease that would interfere with completion of the study or follow-up assessments, or that would adversely affect outcomes

TLC = Total lung capacity; $\mathrm{PaCO}_{2}=$ arterial carbon dioxide tension; $\mathrm{DLCO}=$ carbon monoxide diffusion capacity; RSVP = right systolic ventricular pressure; $\mathrm{LVEF}=$ left ventricular ejection fraction.

Post hoc CT Scan Analyses

In all four trials, the distribution of emphysema on the chest CT scan was based on the treating physicians' own visual judgment. To assess whether the degree of homogeneity impacted the response to coil therapy for $\mathrm{FEV}_{1}, \mathrm{RV}, 6 \mathrm{MWD}$, and SGRQ score, we performed a standardized, qualitative, visual post hoc analysis of the baseline chest CT scans of all patients who had at least a 6-month follow-up visit. Each upper and each lower lobe was scored for severity of emphysema using a 0-5 lung qualitative score. In brief: $0=$ no visible parenchymal damage; $1=$ distributed mild centrilobular damage measuring $1-3 \mathrm{~mm}$ in diameter; $2=$ more pronounced centrilobular damage measuring $5-10 \mathrm{~mm}$ in 
Fig. 2. Patient flowchart.

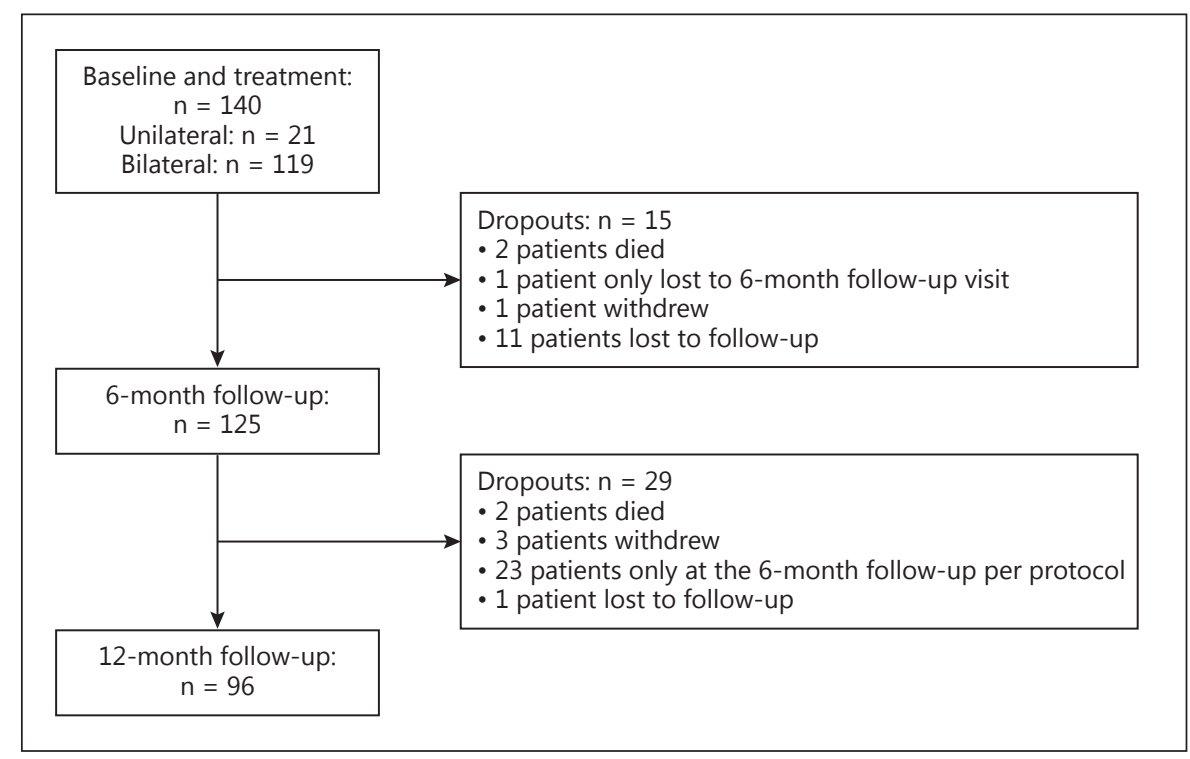

Table 2. Baseline characteristics $(\mathrm{n}=140)$

\begin{tabular}{lc}
\hline Male/female, $\mathrm{n}$ & $64 / 76$ \\
Age, years & $60.68 \pm 7.87$ \\
BMI & $24.66 \pm 4.61$ \\
Smoking, pack-years & $39.46 \pm 21.07^{1}$ \\
\hline FEV $_{1}, 1$ & $0.77 \pm 0.22^{1}$ \\
FEV $_{1}, \%$ predicted & $28.5 \pm 6.9^{2}$ \\
FVC, 1 & $2.56 \pm 0.72^{1}$ \\
FEV $/$ FVC, \% & 31.081 \\
RV, 1 & $5.0(3.2-9.1)$ \\
RV, \% predicted & $233(155-455)$ \\
TLC, 1 & $7.92 \pm 1.43$ \\
RV/TLC ratio & $0.65 \pm 0.08$ \\
DLCO, \% predicted & $32.0(15.4-68.0)^{3}$ \\
\hline SGRQ total score & $60.2 \pm 13.4$ \\
6MWD, m & $316 \pm 100$ \\
mMRC score & $3(2-4)$ \\
\hline Number of patients/study & \\
Slebos [2], 2012 & $25(17.9)$ \\
Shah [7], 2013; Zoumot [10], 2015 & $45(32.1)$ \\
Deslee [8], 2014 & $60(42.9)$ \\
Klooster [9], 2014 & $10(7.1)$ \\
\end{tabular}

Data are presented as $\mathrm{n}(\%)$, mean $\pm \mathrm{SD}$, or median (range). $\mathrm{BMI}=$ Body mass index; FVC $=$ forced vital capacity; $\mathrm{TLC}=$ total lung capacity; DLCO = carbon monoxide diffusion capacity. ${ }^{1} \mathrm{n}=$ 139. ${ }^{2} \mathrm{n}=138 .{ }^{3} \mathrm{n}=125$.

diameter; 3 = blebs and/or small bullae measuring 10-20 $\mathrm{mm}$ in diameter; $4=$ more significant panlobular lung damage that includes larger bullae measuring $20-50 \mathrm{~mm}$ in diameter, and $5=$ severe lung damage that presents with a very compromised lung structure, including lobar damage in which bullae exceed $>50 \mathrm{~mm}$ in diameter and/or lobes that present with very little visible remaining lung structure. The upper lobe score was compared to the lower lobe score in each lung. If the difference in scores between the upper and the lower lobe was 1 or 0 , the lung was considered to be homogeneous. A point difference of $\geq 2$ indicated heterogeneous destruction in the lung. If both lungs were homogeneous, the patient was considered homogeneous. If one or both lungs were considered heterogeneous, the patient was considered to be heterogeneous.

\section{Statistics}

To compare the baseline and follow-up measurements, we performed a paired-samples $t$ test. Missing values were not imputed. We also measured the responder rate using the known MCID for $\mathrm{FEV}_{1}$ [3], RV [4], 6MWD [5], and SGRQ score [6]. To investigate if we could identify independent predictors of change in RV, 6MWD, and SGRQ score at the 6-month follow-up, we performed multiple linear regression analyses. Variables were selected in the model if the $\mathrm{p}$ value was $<0.2$ in univariate analysis. $\mathrm{p}$ values $<0.05$ were considered statistically significant. Statistical analyses were performed using IBM-SPSS statistics version 22 (IBM Corp., Armonk, N.Y., USA). All data used in this trial were independently monitored by a contract research organization.

\section{Results}

\section{Patients}

A total of 140 patients with severe COPD were enrolled in the four studies (see table 2 for the baseline characteristics), and all were included in this meta-analysis. At 6 months, 125 patients had evaluable data, and at 12 months, 96 patients (see fig. 2 for the patient flowchart). 


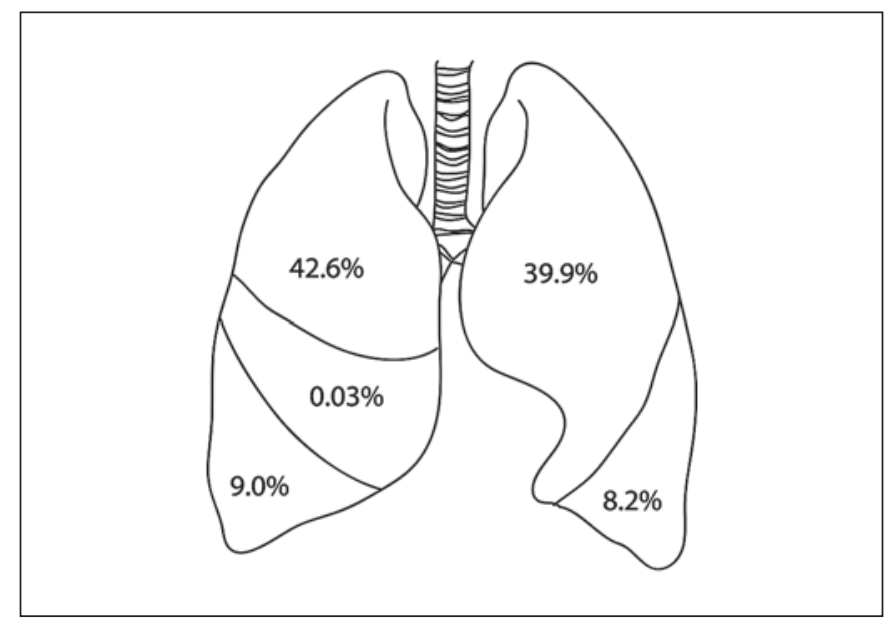

Fig. 3. Distribution per lobe of the 2,536 coils placed in all studies.

\section{The Coil Procedure}

In 140 patients, a total of 259 coil procedures were performed in which 2,536 coils were placed. Table 3 summarizes the procedural characteristics. Figure 3 shows the distribution of the coils per lobe.

\section{Safety}

No periprocedural serious adverse events occurred in any of the 259 coil procedures; no deaths or respiratory failures were reported. Five patients died within 1 year after the treatment, but none were judged to be directly related to the treatment (the causes of death are reported in table 4). The overall rate of pneumothorax requiring a chest tube was $3.5 \%$ on a per-procedure basis or $6.4 \%$ per patient treated. A total of 37 severe COPD exacerbations and 27 pneumonias requiring hospitalization were recorded among all patients up to 1 year of follow-up. The incidence of severe COPD exacerbations was 3.1\% during the 0 -to-30-day posttreatment period, $2.9 \%$ per month during the 30 -to-180-day period, and $2.3 \%$ per month during the 6-month-to-1-year period. The incidence of pneumonia, scored as a serious adverse event, was 3.5\% per month during the 0 -to- 30 -day period, $1 \%$ during the 30 -to-180-day period, and $2.1 \%$ per month during the 6-month-to-1-year posttreatment period. A summary of all respiratory adverse events is shown in table 4 .

\section{Efficacy}

Six and 12 months after coil treatment, significant (all $\mathrm{p}<0.001$ ) improvements were observed in spirometry, lung volume, 6MWD, and quality of life measurements.
Table 3. Coil procedure statistics (259 procedures in 140 patients)

$\begin{array}{lc}\text { Treatment } & \\ \quad \text { Unilateral } & 21(15) \\ \text { Bilateral } & 119(85) \\ \text { Procedure time, min } & 38.5(22-125) \\ \text { Coils per procedure } & 10(6.5-15) \\ \text { Hospital stay, days } & 2(0-40) \\ \text { Coil sizes used } & \\ 70 \mathrm{~mm} & 5(0.2) \\ 85 \mathrm{~mm} & 21(0.83) \\ 100 \mathrm{~mm} & 974(38.4) \\ 125 \mathrm{~mm} & 1,064(42.0) \\ 150 \mathrm{~mm} & 399(15.7) \\ 175 \mathrm{~mm} & 71(2.8) \\ 200 \mathrm{~mm} & 2(0.08)\end{array}$

Data are presented as $\mathrm{n}(\%)$ or median (range).
No change was observed in diffusion capacity (see table 5 for detailed efficacy data). The number of patients responding beyond the MCID for lung volume, spirometry, SGRQ score, and 6MWD are shown in table 6a for both the 6-month and the 12-month follow-up.

\section{Responder Analysis}

Coil treatment seems to provide a higher magnitude of benefit (in SGRQ score, as well as trends for pulmonary function and exercise capacity) when applied bilaterally and when treatment occurs in the upper lobes. No differences in outcome were observed for heterogeneous emphysema patients when compared with homogeneous patients (fig. 4). Furthermore, no association was found between the number of coils placed and efficacy outcomes.

To test for agreement between individual patients reaching the MCIDs for $\mathrm{FEV}_{1}, \mathrm{RV}, 6 \mathrm{MWD}$, and SGRQ score, we observed as expected a high correlation between patients reaching the MCIDs for both RV and FEV ${ }_{1}$. Approximately $60 \%$ of the patients reaching the MCID for $6 \mathrm{MWD}$ also reached the MCIDs for RV, $\mathrm{FEV}_{1}$, and SGRQ score, all indicating a strong correlation between pulmonary functional improvement and the patients' measured outcomes (table 6b).

The multiple linear regression analysis showed that a higher RV at baseline was the only independent predictor of a larger decrease in RV between baseline and the 6-month follow-up. No other independent predictors were found. Lower age and lower 6MWD at baseline were the only independent predictors of a larger im- 
Table 4. Adverse events

\begin{tabular}{|c|c|c|c|c|c|c|c|c|c|}
\hline & \multicolumn{3}{|c|}{$\begin{array}{l}<30 \text { days }^{\mathrm{a}} \\
(\mathrm{n}=140,259 \text { procedures })\end{array}$} & \multicolumn{3}{|c|}{$\begin{array}{l}30-180 \text { days } \\
(\mathrm{n}=125)\end{array}$} & \multicolumn{3}{|c|}{$\begin{array}{l}180-360 \text { days } \\
(\mathrm{n}=96)\end{array}$} \\
\hline & mild & severe & patients & mild & severe & patients & mild & severe & patients \\
\hline Chest pain & 43 & 3 & 36 & 9 & 5 & 13 & 3 & 0 & 3 \\
\hline Hemoptysis & 96 & 0 & 59 & 6 & 0 & 6 & 2 & 0 & 2 \\
\hline Pneumothorax & $12^{\mathrm{b}}$ & 6 & 18 & $1^{\mathrm{b}}$ & 3 & 4 & $1^{\mathrm{b}}$ & 0 & 1 \\
\hline Pneumonia & 10 & 9 & 15 & 7 & 6 & 12 & 3 & 12 & 14 \\
\hline Influenza & 3 & 1 & 4 & 0 & 0 & 0 & 1 & 0 & 0 \\
\hline Dyspnea & 17 & 1 & 15 & 18 & 6 & 20 & 0 & 0 & 0 \\
\hline Death $^{c}$ & - & 0 & 0 & - & 2 & 2 & - & 3 & 3 \\
\hline
\end{tabular}

Events are given as actual numbers scored and number of patients who experienced at least 1 adverse event episode. The incidence rate per month mentioned in the text is calculated by: number of severe adverse events divided by the number of cases, divided by the number of months.

a The entries under '<30 days' show the events scored for the first 30 days after bronchoscopy. Most patients (125 out of 140) were bilaterally treated, therefore the adverse events shown were reported during 2 time periods of 30 days and include 259 cases in total. ${ }^{\mathrm{b}}$ Small pneumothorax requiring no intervention. ${ }^{\mathrm{c}}$ Causes of death: (1) 1 patient developed a severe infective exacerbation of the airways disease secondary to influenza $\mathrm{B}$ and died following a hemorrhagic stroke while ventilated in the intensive care unit; (2) 1 patient developed a severe infective exacerbation of his COPD approximately 10 weeks after his second treatment and died from respiratory failure refractory to ventilatory therapy via endotracheal tube at the end of a 2-week hospital stay; (3) 1 patient died secondary to a severe infective exacerbation of COPD 7 months after his final treatment; (4) 1 patient died from severe urinary sepsis 335 days after coil treatment, and (5) 1 patient died from esophageal cancer 309 days after the second coil treatment. provement in 6MWD between baseline and the 6-month follow-up. Higher SGRQ total score at baseline was the only independent predictor of larger improvement in SGRQ total score between baseline and the 6-month follow-up.

\section{Discussion}

Novel approaches continue to be developed for inducing a volume reduction in patients with emphysema [16]. The approaches employed have ranged from insertion of endobronchial valves [17] and instillation of profibrotic agents $[18,19]$ to creation of artificial air passages [20]. Bronchoscopically placed nitinol coils have shown promising clinical benefits, with particular improvements in patient-centered outcomes such as quality of life and exercise capacity. However, the criticisms of the studies performed thus far have been the small size of the studies and their limited follow-up periods, particularly the time point when the primary endpoints have been evaluated. The studies have all been open-label studies, and only one of the studies was randomized - but only up to the 3-month time point. The aggregated data now address some of these limitations, particularly the number of patients evaluated and the duration of follow-up. This larger data set including patients treated at 13 different institutions in four European countries demonstrates consistent improvements in $\mathrm{FEV}_{1}$, forced vital capacity, RV, 6MWD, SGRQ score, and mMRC score at both the 6 -month and the 12-month time point. The magnitude of improvements is similar in all the studies, and the response rates, based on meaningful clinical criteria, are also similar across the studies.

The current treatment possibilities are of limited benefit to very severe emphysema patients. Effective therapies such as lung volume reduction surgery and lung transplantation do exist but are scarcely used due to limited availability and significant morbidity [21]. The introduction of bronchoscopic techniques for this purpose may serve a great need in a large patient group. The current data support the promise of bronchoscopic coils as a minimally invasive alternative, with a median hospital stay of 2 days, and as a still effective therapy for emphysema that can be used in a significantly larger population of patients than can be served by more invasive techniques. 
Table 5. Absolute change in clinical characteristics between baseline and the 6 -month $(\mathrm{n}=125)$ or the 12 -month follow-up $(\mathrm{n}=95)$

\begin{tabular}{lcccc}
\hline & $\Delta 6$-month FU-BL & p value & $\Delta$ 12-month FU-BL & p value \\
\hline$\Delta$ RV, 1 & $-0.51 \pm 0.85$ & $<0.001$ & $-0.43 \pm 0.72$ & $<0.001$ \\
$\Delta$ TLC, 1 & $-0.26 \pm 0.69$ & $<0.001$ & $-0.22 \pm 0.55$ & $<0.001$ \\
$\Delta$ RV/TLC ratio & $-0.04 \pm 0.07$ & $<0.001$ & $-0.04 \pm 0.06$ & $<0.001$ \\
\hline$\Delta$ FEV $_{1}, 1$ & $0.08 \pm 0.19$ & $<0.001$ & $0.08 \pm 0.21$ & 0.001 \\
$\Delta$ FVC, & $0.22 \pm 0.48$ & $<0.001$ & $0.22 \pm 0.43$ & $<0.001$ \\
\hline$\Delta$ DLCO, \% predicted & $-0.61 \pm 7.8$ & 0.440 & $0.01 \pm 8.1$ & 0.993 \\
\hline$\Delta$ SGRQ symptom score & $-6.3 \pm 24.4$ & 0.005 & $-3.7 \pm 24.6$ & 0.150 \\
$\Delta$ SGRQ activities score & $-8.4 \pm 16.5$ & $<0.001$ & $-8.2 \pm 17.7$ & $<0.001$ \\
$\Delta$ SGRQ impact score & $-11.1 \pm 16.4$ & $<0.001$ & $-8.5 \pm 15.6$ & $<0.001$ \\
$\Delta$ SGRQ total score & $-9.5 \pm 14.3$ & $<0.001$ & $-7.7 \pm 14.2$ & $<0.001$ \\
\hline$\Delta$ 6MWD, m & $44.1 \pm 69.8$ & $<0.001$ & $38.1 \pm 71.9$ & $<0.001$ \\
$\Delta$ mMRC score & $-0.56 \pm 1.05$ & $<0.001$ & $-0.53 \pm 0.86$ & $<0.001$ \\
\hline
\end{tabular}

Data are presented as mean \pm SD. Significant $\mathrm{p}$ values are set in italics. FU = Follow-up; BL = baseline; TLC = total lung capacity; FVC = forced vital capacity; DLCO = carbon monoxide diffusion capacity.

Table 6. Minimal clinically important difference

a Number of patients who reached a minimal clinically important difference at the 6-month and the 12-month follow-up

\begin{tabular}{lll}
\hline & 6 -m FU & $12-\mathrm{m} \mathrm{FU}$ \\
\hline$\Delta \mathrm{RV}>-0.40$ liters [4] & $65(52)$ & $46(48)$ \\
$\Delta \mathrm{RV} / \mathrm{TLC}>-4 \%[4]$ & $62(50)$ & $42(44)$ \\
$\Delta \mathrm{FEV} \mathrm{I}_{1}>0.1$ liters [3] & $48(40)$ & $33(35)$ \\
$\Delta \mathrm{SGRQ}$ total score $>-4$ points [6] & $76(61)$ & $56(59)$ \\
$\Delta 6 \mathrm{MWD}>26 \mathrm{~m}[5]$ & $76(63)$ & $53(57)$ \\
\hline
\end{tabular}

b Number of patients who reached both MCIDs at the 6-month or the 12-month follow-up

\begin{tabular}{|c|c|c|c|c|c|c|}
\hline & \multicolumn{2}{|c|}{$\Delta \mathrm{FEV}_{1}>0.1$ liters } & \multicolumn{2}{|c|}{$\Delta$ SGRQ score $>-4$ points } & \multicolumn{2}{|c|}{$\triangle 6 \mathrm{MWD}>26 \mathrm{~m}$} \\
\hline & 6-m FU & $12-\mathrm{m} \mathrm{FU}$ & 6-m FU & $12-\mathrm{m} \mathrm{FU}$ & 6-m FU & 12-m FU \\
\hline$\Delta \mathrm{RV}>-0.40$ liters & $85(70)$ & $68(72)$ & $78(62)$ & $31(33)$ & $65(54)$ & $43(47)$ \\
\hline$\Delta \mathrm{FEV}_{1}>0.1$ liters & & & $64(53)$ & $44(47)$ & $67(57)$ & $56(61)$ \\
\hline$\Delta$ SGRQ total score $>-4$ points & & & & & $79(65)$ & $61(66)$ \\
\hline
\end{tabular}

Data are presented as $\mathrm{n}(\%)$. Numbers in square brackets behind variables in table 6 a are references supporting the MCID cutoffs. 6-/12-m FU =6-/12-month follow-up; TLC = total lung capacity.

Formal software-based scoring of all the CT scans of the patients who participated in the RePneu coil clinical studies demonstrated that more than half of the patients recruited into the clinical studies had a homogeneous distribution of emphysema. Whereas lung volume reduction surgery only showed a significant benefit in upper lobepredominant disease [22], the coil technique is the first lung volume reduction treatment to demonstrate efficacy in all patients with emphysema, irrespective of the distribution of the disease and regardless of whether the disease 
Fig. 4. Differences between groups in change in clinical characteristics between baseline and 6-month follow-up. Patients who were treated in both lower and upper lobes were not included in the analyses. $* \mathrm{p}<0.05$.

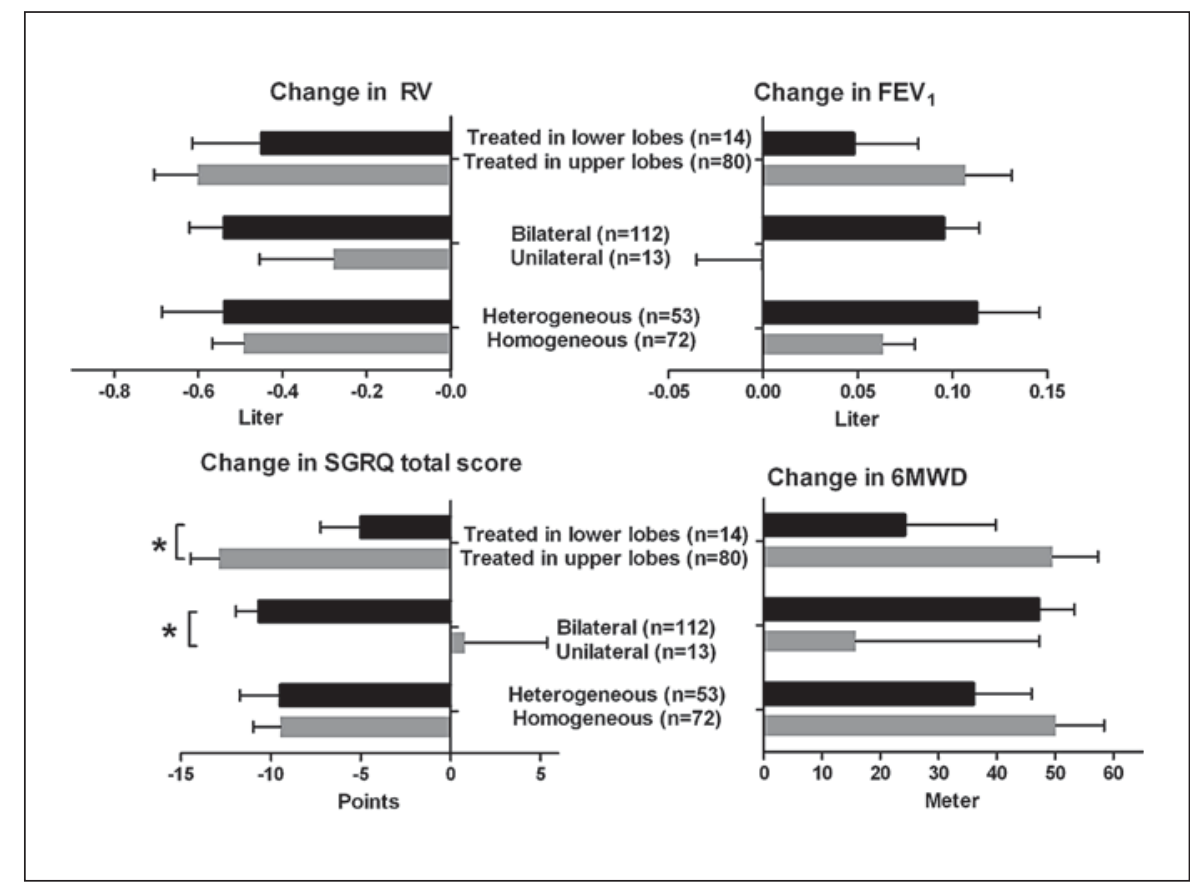

distribution was assessed visually or using standard CT scoring software. An airway bypass, where artificial airways are created to facilitate the escape of trapped gas, was the only treatment strategy specifically designed to treat homogeneous disease [20], but the treatment effects subsided within 1 month. Endobronchial coil treatment has shown a sustained benefit in homogenous patients through to 1 year.

The RePneu endobronchial coil is designed to compress the areas of lung parenchyma most damaged by emphysema in both lungs. Because this shape memory nitinol coil acts by a simple mechanical action, the effects are achieved immediately and independently of collateral ventilation. The proposed coil treatment mechanism of action can be only partially explained by the actual lung volume reduction, as has been shown in our current results demonstrating changes in static lung hyperinflation (RV and RV/total lung capacity ratio). One of the trials in our meta-analysis demonstrated a significant reduction in airway resistance [9]. The induced volume reduction, combined with beneficial changes in lung compliance, can also result in improved diaphragm efficiency. Theoretically, these improvements may also impact a ventilation-perfusion ratio mismatch; however, the fact that we did not observe a change in diffusion capacity might imply that there was no significant change in this mechanism.

Coil Therapy for Emphysema
Further insight into the 'coil physiology' might also help in defining a responder profile to better understand which patients benefit from the treatment and which do not. In the aggregate, our data show a clinically significant response 1 year after coil treatment in $50 \%$ of the patients treated for RV and in $60 \%$ of the patients treated for SGRQ score and 6MWD. A better understanding of predictors of outcome could improve responder rates and avoid subjecting patients to an intervention of limited benefit. However, since COPD is phenotypically a very heterogeneous disease, subtle differences in disease might also contribute to differences in treatment outcome.

The 259 bronchoscopies in the 140 patients included in our meta-analysis showed that in a stable clinical condition, our severe emphysema patients could undergo a 45-min bronchoscopic treatment under general anesthesia or moderate sedation without serious procedural safety issues. Directly after the coil treatment, a pneumothorax for which a chest tube was necessary occurred in up to $6 \%$ of the patients treated, all recovering without surgical intervention. Both COPD exacerbations and pneumonias were observed after treatment, with a predominance of these events in the first 30 days, which was potentially related to the treatment and/or the bronchoscopies and intubations. On average, $2.25 \mathrm{~m}$ of nitinol wire is implanted in the airways of each patient; this can result in some 
local airway irritation, subtle regional airway closures, and tension-induced inflammatory opacities as observed on the posttreatment X-rays in the treated areas. In the clinical trials evaluated in this study, the follow-up time after treatment was from 3 months to a maximum of 1 year. A longer-term follow-up until 3 years after coil treatment has recently been reported for 33 patients also included in this meta-analysis (all Dutch patients who participated in the studies by Slebos et al. [2] and Deslee et al. [8]), showing no unexpected device-related events or excess of exacerbations or pneumonias and no coil migrations [23].

A disadvantage of combining the four different studies was that the CT scans were not performed according to identical scanning protocols. Therefore, the scans could be used for individual scoring of heterogeneity but not for aggregate analysis.

Future directions for the further development of this therapy will have to involve larger multicenter randomized controlled trials, such as the two studies REVOLENS (NCT01822795 France [24]) and RENEW (NCT01608490 USA/EUROPE/Canada), which completed enrollment, and longer follow-up periods assessing safety and effica- cy, as well as gaining more in-depth knowledge of the mechanisms of action.

In conclusion, this meta-analysis was able to confirm the conclusions from the individual trials showing that bronchoscopic coil treatment improves pulmonary function, 6MWD, and quality of life in patients with severe emphysema. We furthermore are able to confirm that these results are durable up to 1 year after treatment, irrespective of whether patients have a heterogeneous or homogeneous distribution of the disease.

\section{Acknowledgements}

The original trials analyzed in this manuscript have all been funded by PneumRx. PneumRx provided all monitored raw study data to the investigators for independent analysis.

\section{Financial Disclosure and Conflicts of Interest}

All authors participated in one of the four clinical trials that have been used for analysis in this manuscript. PneumRx reimbursed trial-related expenses for those trials and provided the study devices used.

\section{References}

-1 Herth FJ, Eberhard R, Gompelmann D, Slebos DJ, Ernst A: Bronchoscopic lung volume reduction with a dedicated coil: a clinical pilot study. Ther Adv Respir Dis 2010;4:225231.

-2 Slebos DJ, Klooster K, Ernst A, Herth FJ, Kerstjens HA: Bronchoscopic lung volume reduction coil treatment of patients with severe heterogeneous emphysema. Chest 2012; 142:574-582.

$\checkmark 3$ Donohue JF: Minimal clinically important differences in COPD lung function. COPD 2005;2:111-124.

4 Hartman JE, Ten Hacken NH, Klooster K, Boezen HM, de Greef MH, Slebos DJ: The minimal important difference for residual volume in patients with severe emphysema. Eur Respir J 2012;40:1137-1141.

$\checkmark 5$ Puhan MA, Chandra D, Mosenifar Z, Ries A, Make B, Hansel NN, Wise RA, Sciurba F; National Emphysema Treatment Trial (NETT) Research Group: The minimal important difference of exercise tests in severe COPD. Eur Respir J 2011;37:784-790.

6 Jones PW: St George's Respiratory Questionnaire: MCID. COPD 2005;2:75-79.
7 Shah P, Zoumot Z, Bicknell S, Ross E, Quiring J, Hopkinson N, Kemp S: Endobronchial coils for the treatment of severe emphysema with hyperinflation (RESET): a randomised controlled trial. Lancet Respir Med 2013;1:233-240.

8 Deslee G, Klooster K, Hetzel M, Stanzel F, Kessler R, Marquette CH, Witt C, Blaas S, Gesierich W, Herth FJ, et al: Lung volume reduction coil treatment for patients with severe emphysema: a European multicentre trial. Thorax 2014;69:980-986.

9 Klooster K, Ten Hacken NH, Franz I, Kerstjens HA, van Rikxoort EM, Slebos DJ: Lung volume reduction coil treatment in chronic obstructive pulmonary disease patients with homogeneous emphysema: a prospective feasibility trial. Respiration 2014;88:116-125.

10 Zoumot Z, Kemp SV, Singh S, Bicknell SR, McNulty WH, Hopkinson NS, Ross ET, Shah PL: Endobronchial coils for severe emphysema are effective up to 12 months following treatment: medium term and cross-over results from a randomised controlled trial. PLoS One 2015; 10:e0122656.

11 Bestall JC, Paul EA, Garrod R, Garnham R, Jones PW, Wedzicha JA: Usefulness of the Medical Research Council (MRC) dyspnoea scale as a measure of disability in patients with chronic obstructive pulmonary disease. Tho$\operatorname{rax} 1999 ; 54: 581-586$.
-12 Miller MR, Hankinson J, Brusasco V, Burgos F, Casaburi R, Coates A, Crapo R, Enright P, van der Grinten CP, Gustafsson P, et al: Standardisation of spirometry. Eur Respir J 2005; 26:319-338.

13 Wanger J, Clausen JL, Coates A, Pedersen OF, Brusasco V, Burgos F, Casaburi R, Crapo R, Enright P, van der Grinten CP, et al: Standardisation of the measurement of lung volumes. Eur Respir J 2005;26:511-522.

-14 ATS Committee on Proficiency Standards for Clinical Pulmonary Function Laboratories: ATS statement: guidelines for the six-minute walk test. Am J Respir Crit Care Med 2002; 166:111-117.

15 Jones PW, Quirk FH, Baveystock CM, Littlejohns P: A self-complete measure of health status for chronic airflow limitation. The St George's Respiratory Questionnaire. Am Rev Respir Dis 1992;145:1321-1327.

16 Mineshita M, Slebos DJ: Bronchoscopic interventions for chronic obstructive pulmonary disease. Respirology 2014;19:1126-1137.

17 Shah PL, Herth FJ: Current status of bronchoscopic lung volume reduction with endobronchial valves. Thorax 2014;69:280-286.

18 Kramer MR, Refaely Y, Maimon N, Rosengarten D, Fruchter O: Bilateral endoscopic sealant lung volume reduction therapy for advanced emphysema. Chest 2012;142:1111-1117. 
19 Snell G, Herth FJ, Hopkins P, Baker KM, Witt C, Gotfried MH, Valipour A, Wagner M, Stanzel F, Egan JJ, et al: Bronchoscopic thermal vapour ablation therapy in the management of heterogeneous emphysema. Eur Respir J 2012;39:1326-1333.

-20 Shah PL, Slebos DJ, Cardoso PF, Cetti E, Voelker K, Levine B, Russell ME, Goldin J, Brown M, Cooper JD, et al: Bronchoscopic lung-volume reduction with Exhale airway stents for emphysema (EASE trial): randomised, sham-controlled, multicentre trial. Lancet 2011;378:997-1005.
21 Vestbo J, Hurd SS, Agustí AG, Jones PW, Vogelmeier C, Anzueto A, Barnes PJ, Fabbri LM, Martinez FJ, Nishimura M, et al: Global strategy for the diagnosis, management, and prevention of chronic obstructive pulmonary disease: GOLD executive summary. Am J Respir Crit Care Med 2013;187:347365.

22 Fishman A, Martinez F, Naunheim K, Piantadosi S, Wise R, Ries A, Weinmann G, Wood DE; National Emphysema Treatment Trial Research Group: A randomized trial comparing lung-volume-reduction surgery with medical therapy for severe emphysema. N Engl J Med 2003;348:2059-2073.
23 Hartman JE, Klooster K, Gortzak K, Ten Hacken NH, Slebos DJ: Long-term followup after bronchoscopic lung volume reduction treatment with coils in patients with severe emphysema. Respirology 2015;20:319326.

24 Deslee G, Barbe C, Bourdin A, Durand-Zaleski I, Dutau H, Jolly D, Jounieaux V, Kessler R, Mal H, Pison C, et al: Cost-effectiveness of lung volume reduction coil treatment in emphysema. STIC REVOLENS (in French). Rev Mal Respir 2012;29:1157-1164. 


\section{Erratum}

Table $6 \mathrm{~b}$ in the paper by Slebos D-J et al. entitled 'Bronchoscopic coil treatment for patients with severe emphysema: a meta-analysis' [Respiration 2015;90:136-145] should read:

Table 6. Minimal clinically important difference

b Number of patients who reached both MCIDs at the 6-month or the 12-month follow-up

\begin{tabular}{|c|c|c|c|c|c|c|}
\hline & \multicolumn{2}{|c|}{$\Delta \mathrm{FEV}_{1}>0.1$ liters } & \multicolumn{2}{|c|}{$\Delta$ SGRQ score $>-4$ points } & \multicolumn{2}{|c|}{$\Delta 6 \mathrm{MWD}>26 \mathrm{~m}$} \\
\hline & 6-m FU & $12-\mathrm{m} \mathrm{FU}$ & 6-m FU & $12-\mathrm{m} \mathrm{FU}$ & 6-m FU & $12-\mathrm{m} \mathrm{FU}$ \\
\hline$\Delta \mathrm{RV}>0.4$ liters & $37(31)$ & $26(28)$ & $47(38)$ & $24(26)$ & $41(34)$ & $24(26)$ \\
\hline$\Delta \mathrm{FEV}_{1}>0.1$ liters & & & $32(26)$ & $20(22)$ & $35(30)$ & $25(27)$ \\
\hline$\Delta$ SGRQ total score $>-4$ points & & & & & $54(45)$ & $38(41)$ \\
\hline
\end{tabular}

Data are presented as $\mathrm{n}(\%)$. Numbers in square brackets behind variables in table 6 a are references supporting the MCID cutoffs. 6-/12-m FU = 6-/12-month follow-up; TLC = total lung capacity. 